\title{
Variations Saisonnières Des Paramètres De Reproduction Et Relation Taille-Poids De Auxis thazard (Lacepède, 1800) Capturé Dans Le Golfe De Guinée Par La Flottille Artisanale Ivoirienne
}

\author{
Edoukou Abekan \\ Laboratoire de Biologie et de Cytologie Animales \\ UFR des Sciences de la Nature, Université Nangui Abrogoua, Côte d'Ivoire \\ Diaha N'guessan Constance \\ Amande Monin Justin \\ Département des Ressources Aquatiques Vivantes \\ Centre de Recherches Océanologiques, Côte d'Ivoire \\ N'guessan Yao \\ Assan N'dri Florentine \\ Angui Kouamé Jean Paul \\ N'da Konan \\ Laboratoire de Biologie et de Cytologie Animales \\ UFR des Sciences de la Nature, Université Nangui Abrogoua, Côte d'Ivoire
}

Doi: 10.19044/esj.2017.v13n33p444 URL:http://dx.doi.org/10.19044/esj.2017.v13n33p444

\begin{abstract}
In this study, the seasonal variation in sex ratio, macroscopic stages of sexual maturity, gonado-somatic ratio, hepatostatic ratio, condition factor, and size-weight relationship in Auxis thazard from Gulf of Guinea were studied from January to December 2016. Sex ratio observed was in favor of males $\left(1: 0,78 ; x^{2}\right.$ observed $=20,16>$ theoretical $\left.x^{2}=3,84 ; p<0,05\right)$. The variation of maturity stages associated with GSI, HSI, and K during different seasons of the year showed that this specie spawns throughout the year. Length-weight relationship was $\mathrm{W}=0,0039 \mathrm{~L}^{3,4108}\left(\mathrm{R}^{2}=0,93 ; \mathrm{N}=291\right)$ for males, $\mathrm{W}=0,0036 \mathrm{~L}^{3,4376}\left(\mathrm{R}^{2}=0,91 ; \mathrm{N}=227\right)$ for females, and $\mathrm{W}=0,004$ $\mathrm{L}^{3,4118}\left(\mathrm{R}^{2}=0,92 ; \mathrm{N}=519\right)$ for the combined sexes. The $b$ value was significantly higher than the theoretical value of 3 for males ( $\mathrm{t}$-test, $\mathrm{t}=$ 233,218, $\mathrm{p}<0,05$ ), for females (t-test, $\mathrm{t}=227,776, \mathrm{p}<0,05$ ), and for all individuals ( $\mathrm{t}$-test, $\mathrm{t}=315,821, \mathrm{p}<0,05$ ). This situation however indicates a faster increase of the fish in weight rather than in length.
\end{abstract}

Keywords: Auxis thazard, seasonal variation, length-weight relationship 


\section{Resume}

Dans cette étude, la variation saisonnière de la sex-ratio, des stades macroscopiques de maturité sexuelle, du rapport gonado-somatique, du rapport hépato-somatique, du facteur de condition et la détermination de la relation taille-poids chez Auxis thazard provenant du golfe de Guinée ont été étudiées de janvier à décembre 2016. La sex-ratio déterminée a montré une prédominance des mâles $\left(1: 0,78 ; x^{2}\right.$ observé $=20,16>x^{2}$ théorique $=$ $3,84 ; \mathrm{p}<0,05)$. Le suivi de la variation des stades de maturité combiné au RGS, RHS et $\mathrm{K}$ au cours des différentes saisons a montré que cette espèce a une reproduction étalée sur toute l'année. La relation taille-poids déterminée pour l'espèce est de $\mathrm{W}=0,0039 \mathrm{~L}^{3,4108}\left(\mathrm{R}^{2}=0,93 ; \mathrm{N}=291\right)$ pour les mâles, $\mathrm{W}=0,0036 \mathrm{~L}^{3,4376}\left(\mathrm{R}^{2}=0,91 ; \mathrm{N}=227\right)$ pour les femelles et $\mathrm{W}=0,004 \mathrm{~L}^{3}$, $4118\left(\mathrm{R}^{2}=0,92 ; \mathrm{N}=519\right)$ pour les deux sexes combinés. Il apparait que l'espèce grossit plus vite qu'elle ne grandit (allométrie positive ou majorante).

Mots clés : Auxis thazard, variation saisonnière, relation taille-poids

\section{Introduction}

Appartenant au groupe des thonidés mineurs et à la famille des Scombridés, l'Auxide (Auxis thazard) est une espèce épipélagique, néritique et océanique présente dans toutes les eaux tropicales et subtropicales du monde (Iccat, 2010). Cette espèce est majoritaire dans les captures de poissons transbordés dans les différents ports des pays africains côtiers bordant le golfe de Guinée. Ces poissons sont de ce fait d'une importance primordiale sur le plan socio-économique pour de nombreuses communautés côtières car leur pêche procure de nombreux emplois (Diaha et al., 2009).

Plusieurs études entreprises sur la biologie de Auxis thazard dans l'océan atlantique ont été par la suite attribuées à Auxis rochei par les mêmes auteurs (Iccat, 2010).

D'autres études sur la reproduction de l'espèce dans les eaux ivoiriennes ont été menées par Bahou et al. (2016), mais n’ont portées que sur la variation mensuelle des paramètres de reproduction des femelles tels le rapport gonado-somatique, le rapport hépato-somatique et le facteur de condition.

Ce travail abordé sur des spécimens provenant du golfe de Guinée a donc pour but de suivre les variations mensuelles et saisonnières de certains paramètres de la reproduction, de déterminer la ou les périodes de ponte et d'établir l'équation de la relation taille-poids. 


\section{Méthodologie d'étude \\ Zone d'étude}

Les spécimens d'auxide ayant servi à notre étude ont été pêchés dans la Zone Economique Exclusive ivoirienne (ZEE) par les pêcheurs artisans. Cette zone marine fait partie du golfe de Guinée qui s'étend depuis la côte sénégalaise $\left(17^{\circ} \mathrm{W}\right)$ jusqu'à l'estuaire du Gabon $\left(10^{\circ} \mathrm{E}\right)$ (Pezennec et al., 1992). Le golfe de Guinée occupe environ 3300 à $3500 \mathrm{~km} \mathrm{de}$ développement côtier et sa façade maritime regroupe des ZEE de plus de 12 pays côtiers. La zone maritime de la Côte d'Ivoire s'étend du cap des Palmes $\left(8^{\circ} \mathrm{W}\right)$ à l'ouest jusqu'au cap des trois Pointes $\left(2^{\circ} 30^{\prime} \mathrm{W}\right)$ à l'est, sur une longueur d'environ 600 kilomètres et une superficie de $200.000 \mathrm{~km}^{2}$ (Abe et al., 2002). Cette zone est caractérisée par deux périodes climatiques : chaude et froide. Les périodes chaudes regroupent deux saisons : La grande saison chaude allant de mars à juin et la petite saison chaude de novembre à décembre (Gouriou, 1988). Les saisons froides qui se caractérisent par des températures comprise entre 21 et $25,5{ }^{\circ} \mathrm{C}$, sont également constituées de deux saisons : La grande saison froide qui part de juillet en octobre et la petite saison froide de janvier à février (Gouriou, 1988). Pendant les saisons chaudes par contre la température est plus élevée, oscillant entre 26 et $30^{\circ} \mathrm{C}$ (Golé Bi et al., 2005).

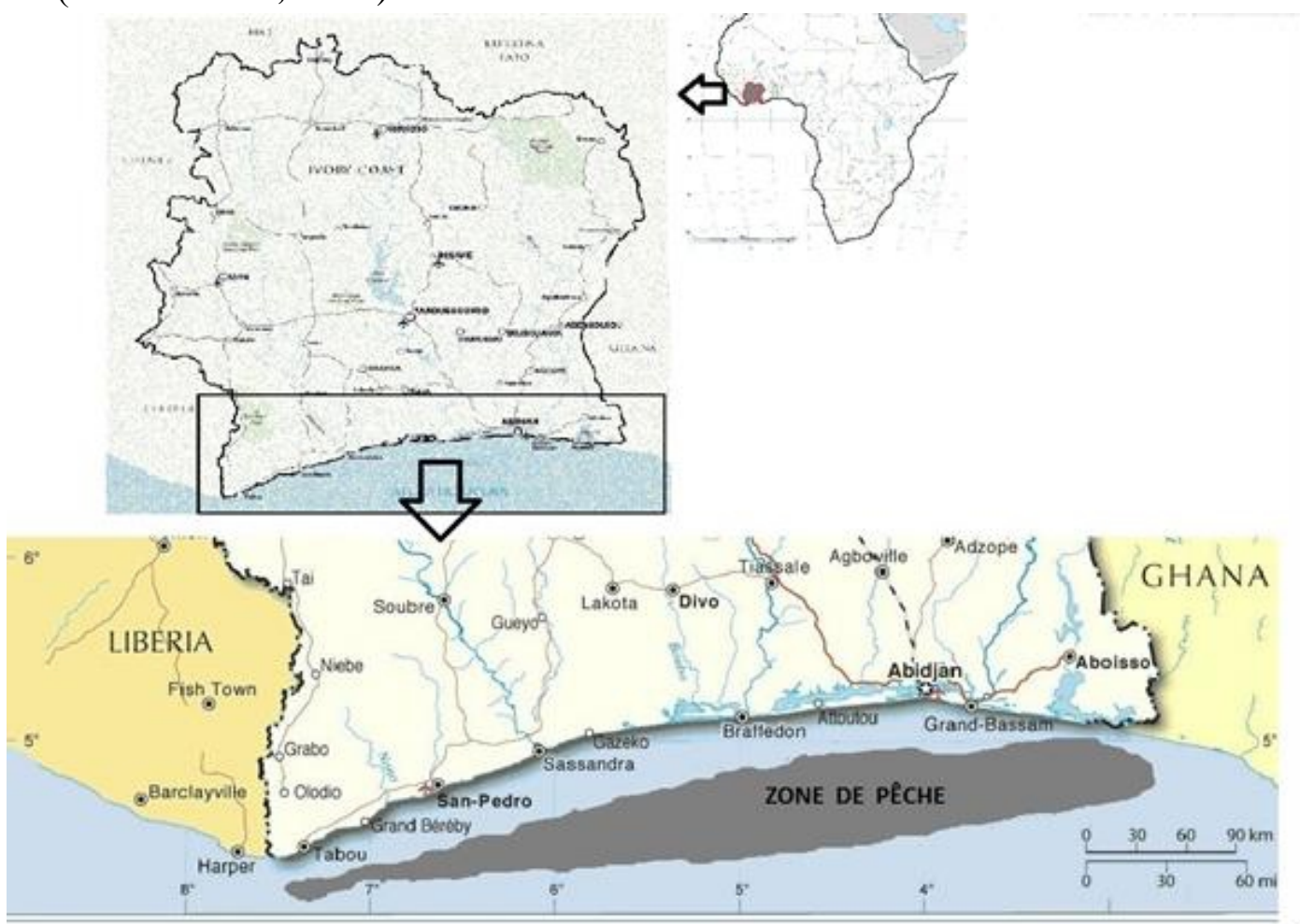

Figure 1. Zone de pêche des pêcheurs artisans ivoiriens exerçant dans le golfe de guinée (Aquamaps, 2016) 


\section{Echantillonnage}

Un échantillonnage basé sur les produits de la pêcherie commerciale est effectué chaque mois au niveau des quais de débarquement des pécheurs artisans situés dans le district d'Abidjan. Il s'agit principalement de la zone de Zimbabwe et celle d'Abobo-doumé. Sur tous les spécimens collectés et transportés au laboratoire, les mensurations suivantes ont été effectuées : La longueur à la fourche $(\mathrm{LF}$ en $\mathrm{cm})$ est mesurée depuis l'extrémité du museau jusqu'au centre de l'échancrure de la nageoire caudale à l'aide d'un ichtyomètre ; le poids (en $\mathrm{Kg}$ ) est obtenu à l'aide d'une balance électronique de $15 \mathrm{~kg}$ de portée et de précision $0,01 \mathrm{~g}$.

Une incision est ensuite effectuée au niveau de la cavité abdominale $\mathrm{du}$ poisson, les viscères et les gonades sont délicatement extraites puis séparées. Après identification du sexe par observation des gonades, le stade macroscopique de maturité sexuelle est déterminé selon l'échelle de Rey et al. (1984). Enfin, les gonades, le foie et l'estomac sont individuellement pesés à l'aide d'une balance électronique.

\section{Stades Macroscopiques de Maturité Sexuelle}

La détermination des stades macroscopiques de maturité sexuelle a été faite par observation directe de la gonade, en s'intéressant à sa coloration, sa consistance, sa vascularisation et le volume qu'elle occupe dans la cavité abdominale. Toutes ces observations ont été faites en se basant sur l'échelle de Rey et al. (1984) qui définit 6 stades aussi bien chez les mâles que chez les femelles.

Tableau 1. Echelle des stades macroscopiques de maturité sexuelle (Rey et al., 1984)

\begin{tabular}{|c|c|c|}
\hline Stades & Mâles & Femelles \\
\hline $\begin{array}{l}\text { Stade } \\
\text { 1.Inderteminés }\end{array}$ & $\begin{array}{l}\text { Sexe indéterminé, gonades en forme de } \\
\text { ruban mince, de couleur pale, collées à la } \\
\text { paroi interne du corps }\end{array}$ & $\begin{array}{l}\text { Sexe indéterminé, gonades en forme de ruban } \\
\text { mince, de couleur pale, collées à la paroi interne } \\
\text { du corps }\end{array}$ \\
\hline $\begin{array}{l}\text { Stade } \\
\text { 2. Immatures }\end{array}$ & $\begin{array}{l}\text { Le sexe identifiable, les gonades minces, } \\
\text { aplatis en forme du ruban de coloration } \\
\text { rose }\end{array}$ & $\begin{array}{l}\text { Le sexe identifiable, les gonades minces, } \\
\text { allongées en forme cylindrique de coloration } \\
\text { rose }\end{array}$ \\
\hline $\begin{array}{l}\text { Stade } \\
\text { 3. Début } \\
\text { maturation } \\
\text { repos sexuel }\end{array}$ & $\begin{array}{l}\text { Les gonades sont de plus grande taille de } \\
\text { couleur blanchâtre }\end{array}$ & $\begin{array}{l}\text { Gonades en forme cylindrique, de coloration } \\
\text { variant de rose obscure au rouge clair. Une } \\
\text { légère vascularisation commence à être visible à } \\
\text { la surface. Les ovocytes ne sont pas visibles à } \\
\text { travers la membrane de l'ovaire ni par incision }\end{array}$ \\
\hline $\begin{array}{l}\text { Stade } \\
\text { 4. Maturation }\end{array}$ & $\begin{array}{l}\text { Gonades très développées, de couleur } \\
\text { blanchâtre. Un peu de sperme s'écoule si } \\
\text { l'on pratique une incision suivi de } \\
\text { pression sur la gonade }\end{array}$ & $\begin{array}{l}\text { Gonades développées de couleur jaune orangé. } \\
\text { Vascularisation très étendue. Les ovocytes sont } \\
\text { visibles par incision ou à travers la paroi de } \\
\text { l'ovaire }\end{array}$ \\
\hline $\begin{array}{l}\text { Stade } \\
\text { 5. Maturation } \\
\text { avancée (Ponte) }\end{array}$ & $\begin{array}{l}\text { Les gonades atteignent leur taille } \\
\text { maximale. Des tâches rouges peuvent } \\
\text { être observées à la surface. Le sperme } \\
\text { s'écoule avec une simple pression }\end{array}$ & $\begin{array}{l}\text { Les gonades ont atteint leur développement } \\
\text { maximum, occupant la totalité de la cavité } \\
\text { abdominale. Les ovocytes sont grosses et } \\
\text { translucides sortant facilement avec une simple }\end{array}$ \\
\hline
\end{tabular}


pression sur l'abdomen

Stade Gonades flasques, contenant encore un

6. Post-ponte

peu du sperme si la ponte est récente. Les gonades sont de coloration rose (ponte très récente) ou plus blanches si la ponte a lieu depuis longtemps

\section{Traitement des Données}

Les informations collectées sont regroupées par mois, par saison et par sexe en se basant sur les subdivisions saisonnières obtenues par Gouriou (1988), en vue de déterminer les différents paramètres tels la sex-ratio, le rapport gonado-somatique, le rapport hépato-somatique, le facteur de condition et la relation taille-poids.

\section{Sex-Ratio (SR)}

La répartition numérique des sexes ou sex-ratio est un indice biologique important car la proportion des mâles et des femelles peut affecter le succès reproductif de l'espèce. Il renseigne sur l'équilibre des sexes à l'intérieur d'une population donnée. Chez l'auxide, le dimorphisme sexuel n'étant pas prononcé, la détermination de la sex-ratio n'est possible qu'après éviscération et détermination macroscopique du sexe par observation directe de la gonade. Elle a été déterminée par mois et par saison. Sa formule est la suivante:

$$
S R=\frac{M}{F}
$$

$M:$ nombre de mâles et $F:$ nombre des femelles.

\section{Rapport Gonado-Somatique (RGS)}

Le rapport gonado-somatique permet d'indiquer le stade de développement des gonades et de déterminer les périodes de ponte au cours du cycle sexuel d'une espèce (West, 1990; Mahé et al., 2005). L'évolution temporelle du RGS d'un poisson mature suit généralement trois phases : une première phase où le RGS commence à augmenter pour atteindre un pic et qui correspond à la phase de maturation, une seconde phase où il diminue correspondant à la ponte, puis une troisième phase où le rapport est minimum, correspondant à la période de repos biologique ou sexuel. Il a été déterminé par mois, par saison et par sexe selon l'expression suivante:

$$
R G S=\frac{P g}{P t} * 100
$$

$P g:$ poids des gonades en $\mathrm{g}$ et $P t:$ poids total du poisson en $\mathrm{g}$. 


\section{Rapport Hépato-Somatique (RHS)}

Chez les poissons, le foie joue un rôle important dans le processus d'élaboration des produits génitaux. Cet organe est responsable de la synthèse de la vitellogénine, principale protéine précurseur du vitellus des œufs (Belhoucine, 2012). Il a été déterminé par mois, par saison et par sexe selon l'équation suivante:

$$
R H S=\frac{P f}{P t} * 100
$$

$P f:$ poids du foie en gramme et $P t$ : poids total du poisson en gramme.

\section{Facteur de Condition (K)}

L'évolution mensuelle du coefficient de condition permet de déduire une stratégie dans l'utilisation des apports énergétiques. En effet, K est corrélé positivement à la densité des lipides. Ces derniers étant utilisés durant les périodes de jeûne, de reproduction et de maturation (Robinson et al., 2008). Il est notamment plus faible après la reproduction. Ce facteur a été déterminé par mois, par saison et par sexe à partir de la formule suivante:

$$
K=\frac{P t}{L f^{3}} * 100
$$

$P t:$ poids total du poisson en get $L f:$ longueur à la fourche en $\mathrm{cm}$.

\section{Relation Taille-Poids}

La relation entre la longueur à la fourche et le poids total des poissons est de type exponentiel. Elle est représentée par la formule décrite par Le Cren (1951) et Ricker (1975):

$$
W=a L^{b}
$$

Avec $W=$ poids total du poisson en gramme, $L=$ longueur à la fourche du poisson en centimètre, $a=$ une constant et $b=$ le taux d'allométrie.

Cette relation, par transformation logarithmique de l'expression précédente peut devenir linéaire afin de réduire la variabilité et d'homogénéiser les deux variables : $W$ et $L$

$$
\log W=\log a+b \log L
$$

Les paramètres $a$ et $b$ ont été calculé par la méthode des moindres carrés. Si $b=3$, la croissance est dite isométrique; $b>3$, indique une meilleure croissance en poids qu'en longueur et $\mathrm{b}<3$ suppose que le poisson grandit plus vite qu'il ne grossit (Micha, 1973; Ricker, 1980). 


\section{Relation Taille-Poids}

La relation entre la longueur à la fourche et le poids total des poissons est de type exponentiel. Elle est représentée par la formule décrite par Le Cren (1951) et Ricker (1975):

$$
W=a L^{b}
$$

Avec $W=$ poids total du poisson en gramme, $L=$ longueur à la fourche du poisson en centimètre, $a=$ une constant et $b=$ le taux d'allométrie.

Cette relation, par transformation logarithmique de l'expression précédente peut devenir linéaire afin de réduire la variabilité et d'homogénéiser les deux variables : $W$ et $L$

$$
\log W=\log a+b \log L
$$

Les paramètres $a$ et $b$ ont été calculé par la méthode des moindres carrés. Si $b=3$, la croissance est dite isométrique ; $b>3$, indique une meilleure croissance en poids qu'en longueur et $\mathrm{b}<3$ suppose que le poisson grandit plus vite qu'il ne grossit (Micha, 1973; Ricker, 1980).

\section{Résultats}

Sex-Ratio

Les spécimens d'auxide provenant du golfe de Guinée échantillonnés de janvier à décembre 2016 sont au nombre de 519 dont 291 mâles $(56,07$ $\%), 227$ femelles $(43,74 \%)$, et 01 individu de sexe indéterminé $(0,19 \%)$. La sex-ratio globale déterminée pour cette espèce étant de 1,28 soit 1 mâle pour 0,78 femelle, est significativement différente de la sex-ratio théorique ( $\chi^{2}$ observé $=20,16>\chi^{2}$ théorique $\left.=3,84 ; \mathrm{p}<0,05\right)$.

\section{Evolution Mensuelle de la Sex-ratio}

La sex-ratio déterminée par mois présente de légères variations en faveur des mâles de janvier à avril $(1: 0,73 ; 1: 0,73 ; 1: 0,86$ et $1: 1,55)$, de septembre à octobre $(1: 0,85 ; 1: 0,78)$ puis s'équilibre en juin $(1: 1)$. Cependant la sex-ratio calculée n'est pas significativement différente de la sex-ratio théorique $1: 1$ ( $\chi^{2}$ observés $<\chi^{2}$ théorique $=3,84 ; p>0,05$ ). La sex-ratio calculée en mai $(1: 0,33)$, août $(1: 0,36)$, novembre et décembre (1:0,33 et $1: 0,33)$ montre une dominance des mâles qui présente une différence significative contrairement au cas précédent ( $\boldsymbol{X}^{2}$ observés $>\boldsymbol{X}^{2}$ théorique $=3,84 ; p<0,05)$. En juillet, ce sont les femelles qui sont dominantes et la sex-ratio est significativement différente de la sex-ratio théorique $\left(1: 1,64 ; x^{2}\right.$ observé $=4,88>x^{2}$ théorique $\left.=3,84 ; \mathrm{p}<0,05\right)$. 


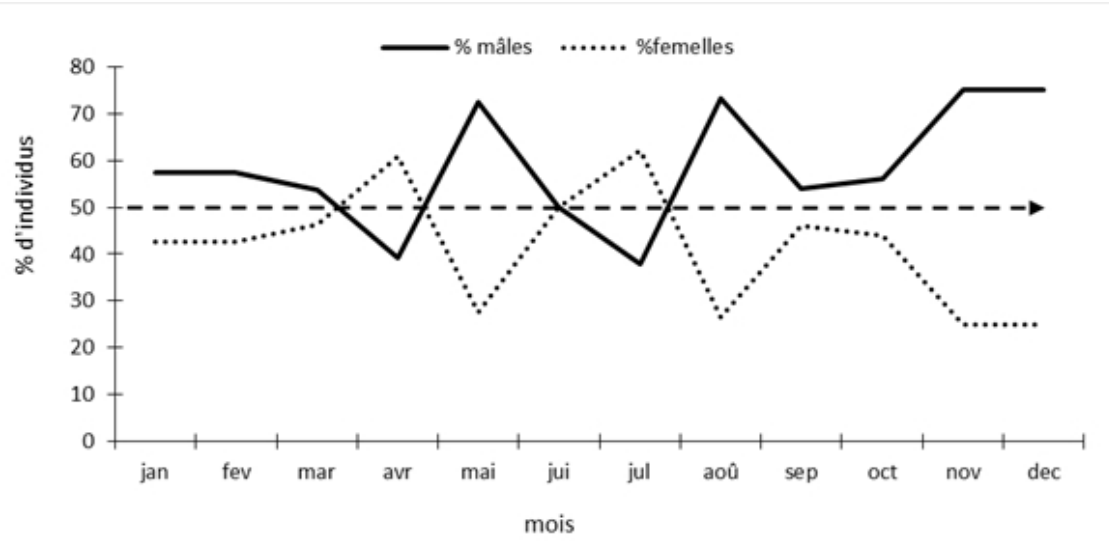

Figure 2. Sex-ratios mensuelles chez Auxis thazard de janvier à décembre 2016 de la pêcherie artisanale ivoirienne

\section{Evolution Saisonnière de la Sex-Ratio}

La sex-ratio enregistrée par saison est en faveur des mâles $(1: 0,73$; $1: 0,83 ; 1: 0,92$ et $1: 0,33)$ sur toute la période d'étude. Toutefois, elle n'est pas significativement différente de la sex-ratio théorique $(1: 1)$ au cours des trois premières saisons (PSF, GSC et GSF) car les Khi-carré observés sont inférieurs au Khi-carré théorique ( $\chi^{2}$ observés $=2,70 ; 1,26$; $0,33<\chi^{2}$ théorique $=3,84$; au seuil de $\left.\mathrm{p}>0,05\right)$. Par contre, pendant la petite saison froide, cette différence est significative $\left(\chi^{2}\right.$ observé $=12>$ $3,84$; au seuil de $\mathrm{p}<0,05)$.

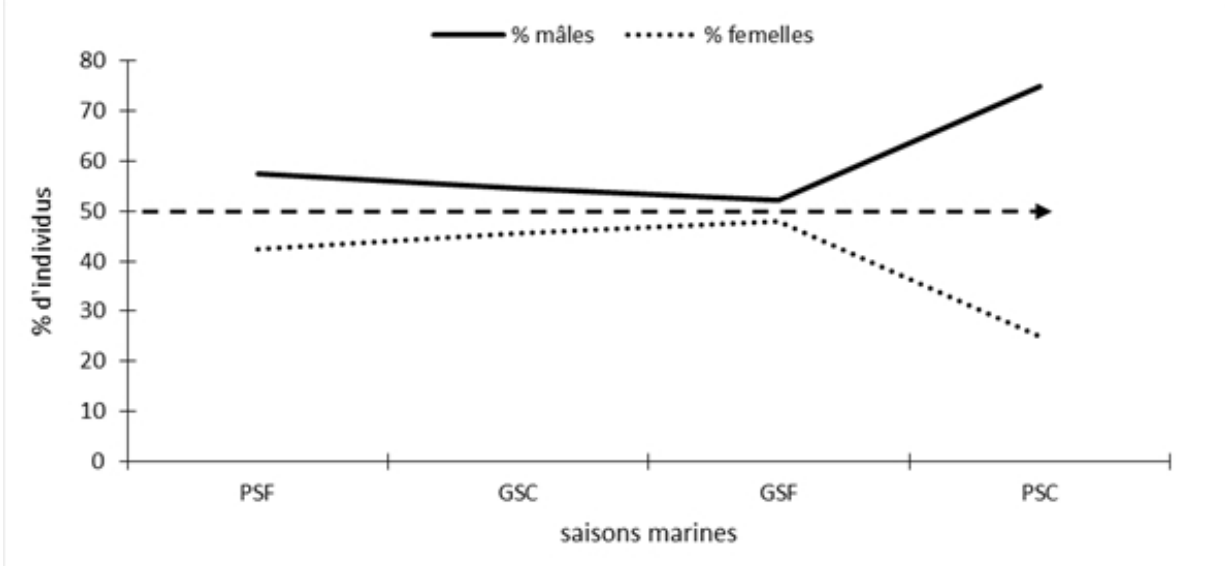

Figure 3. Sex-ratios saisonnières chez Auxis thazard de janvier à décembre 2016 de la pêcherie artisanale ivoirienne

\section{Distribution Mensuelle des Sexes}

Les mâles sont majoritaires dans la plupart des mois, excepté les mois d'avril ( 09 mâles ; 14 femelles) et de juillet ( 31 mâles ; 51 femelles) où les femelles sont nombreuses. Aussi, le mois de juin enregistre autant de 
mâles que de femelles (12 mâles et 12 femelles). Sur toute l'année, les mois de février et de juillet enregistrent respectivement le plus grand nombre de mâles (46) et de femelles (51).

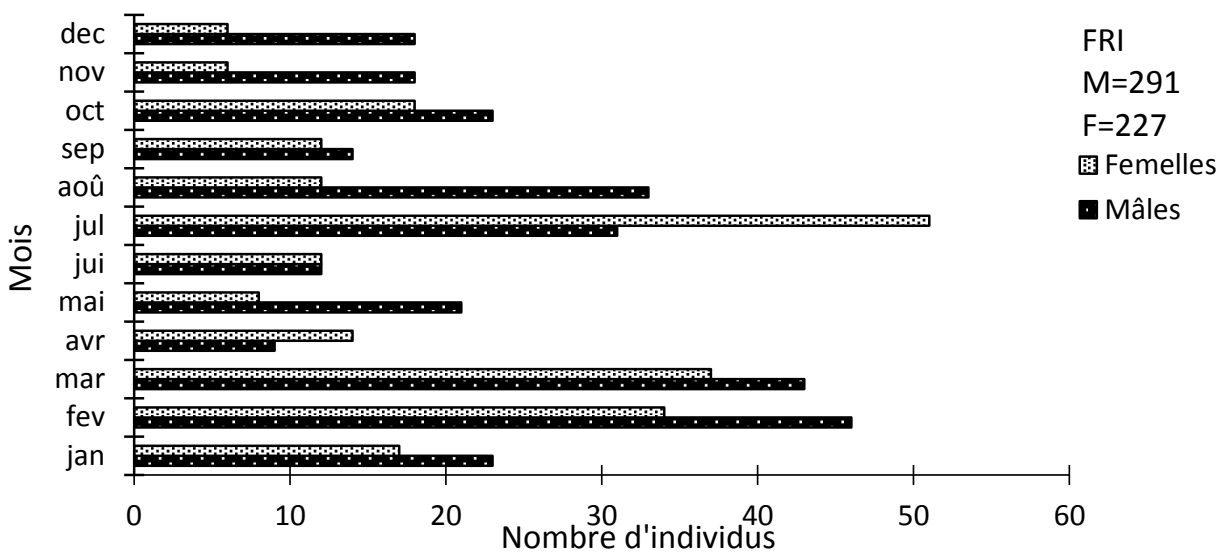

Figure 4. Evolution mensuelle des sexes chez Auxis thazard de la pêcherie artisanale ivoirienne de janvier à décembre 2016

\section{Distribution Saisonnière des Sexes}

Le nombre de femelles enregistré est inférieur à celui des mâles au cours des quatre saisons marines. La grande saison froide a enregistré le plus grand nombre d'individus aussi bien chez les mâles que chez les femelles avec des effectifs respectifs de 101 et de 93. La petite saison chaude par contre totalise les plus faibles valeurs de spécimens échantillonnés au niveau des deux sexes avec 12 individus pour les mâles et 36 pour les femelles.

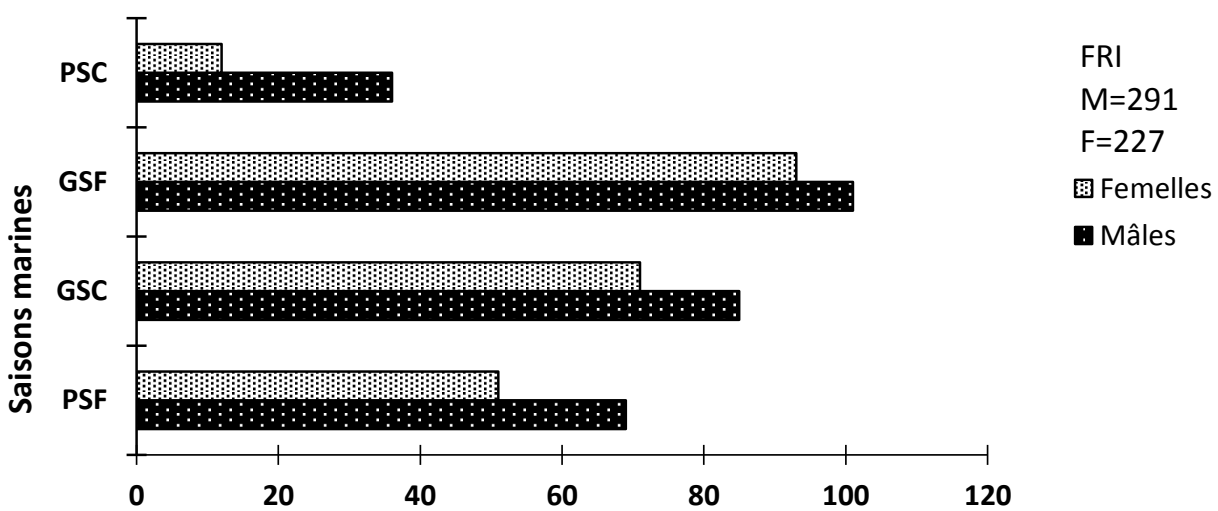

Nombre d'individus

Figure 5. Evolution des sexes chez l'auxide en fonction des saisons marines. 


\section{Evolution des Stades de Maturité Sexuelle}

L'étude des stades de maturité sexuelle des auxides a permis de mettre en exergue l'évolution de la maturation de ces poissons au cours des mois et des saisons. La figure 6 montre les fréquences des stades macroscopiques rencontrés respectivement chez les mâles (FRI-M) et les femelles (FRI-F) échantillonnés. Chez l'auxide (mâles et femelles), tous les stades macroscopiques de maturité sexuelle ont été observés avec une dominance des stades de maturité avancés (III, IV, V, VI) totalisant 90,18\% de l'ensemble de l'échantillon. Au niveau des mâles, le stade IV est dominant (30,24 \% des individus) avec un pic en mars. Les spécimens mâles rencontrés pendant cette période sont en majorité au stade mature avec 87,12 $\%$. Les immatures quant à eux sont faiblement représentés et totalisent 12,71 $\%$ des mâles. Pour les femelles, le stade VI (post ponte) est majoritairement représenté suivi du stade $\mathrm{V}$ (ponte) avec des proportions respectives de 45,81 $\%$ et de $23,35 \%$. Les individus du stade II (immatures) sont faiblement représentés à raison de 5,73\% de l'effectif total des femelles.
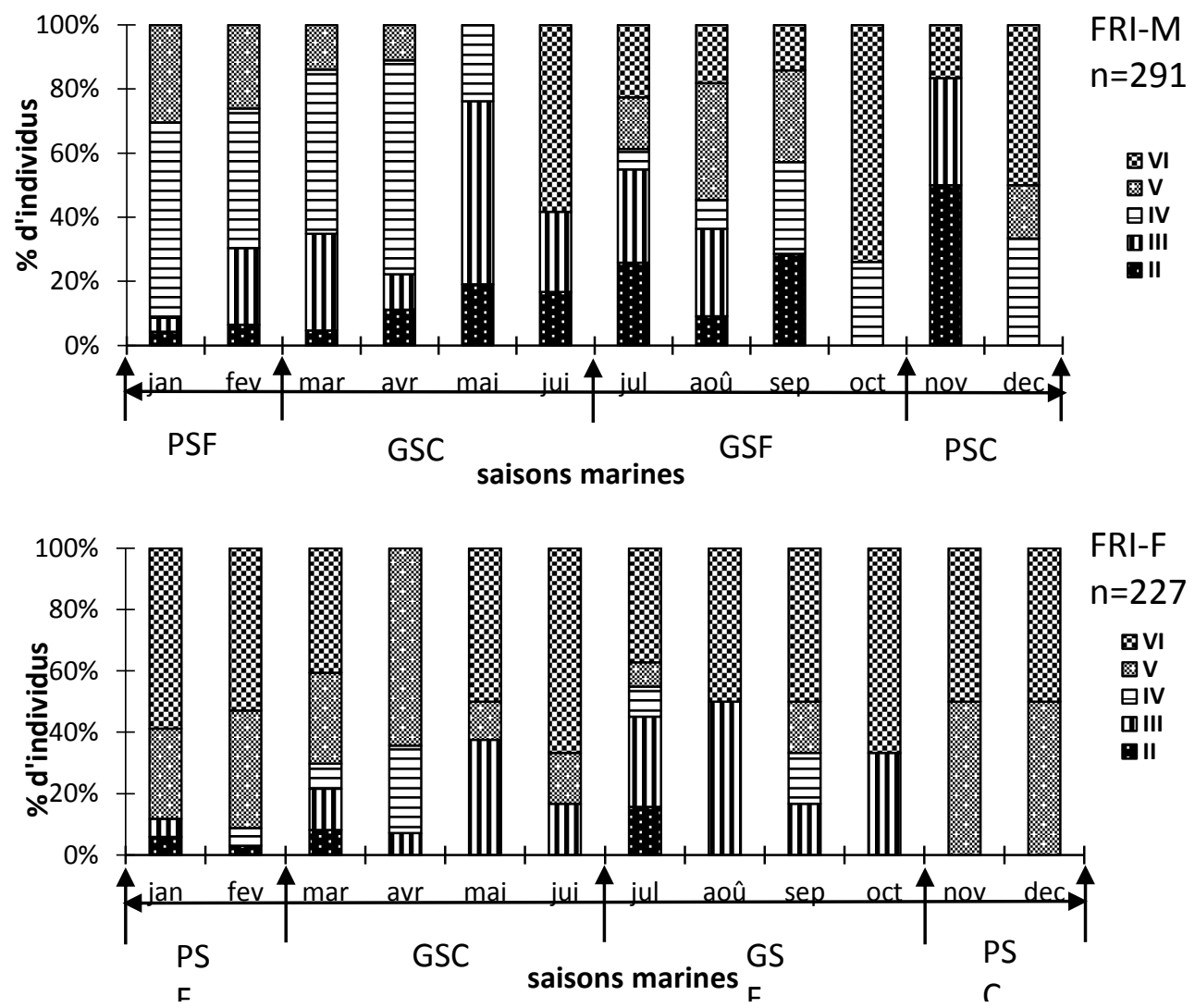

Figure 6. Evolution saisonnière des stades macroscopiques de maturité sexuelle chez l'auxide mâle (FRI-M), femelle (FRI-F) de janvier à décembre 2016 


\section{Rapports Gonado-Somatiques}

L'évolution des RGS chez les mâles et chez les femelles présente la même allure. Elles évoluent généralement en dents de scie tout au long de la période d'étude. Pour les femelles, le RGS décroît de janvier à août puis croît jusqu'en décembre. Plusieurs pics sont observés au cours des quatre saisons dont les principaux en petite saisons froide (janvier) et chaude (décembre) avec des valeurs respectives de 4,97 $\pm 2,102$ et de 4,88 $\pm 1,257$. La plus petite valeur de RGS au niveau des femelles est observée pendant le mois d'août pour une valeur de $1,37 \pm 0,823$. Le test HSD de Tukey montre une différence significative (ANOVA, $\mathrm{p}<0,05$ ) entre les valeurs du RGS femelle de la petite saison froide et celles des grandes saisons chaude et froide. Le RGS mâle, par contre décroît de janvier à août puis augmente en septembre où il atteint un pic $(2,91 \pm 1,991)$, avant de chuter en novembre pour ensuite augmenter légèrement en décembre. La plus faible valeur de RGS mâle est observée dans le mois de novembre avec une valeur de 1,02 \pm 0,807. Le test HSD de Tukey appliqué au niveau des RGS mâles pour les saisons affiche une différence significative (ANOVA, $p<0,05$ ) au cours de la grande saison froide et de la petite saison chaude.

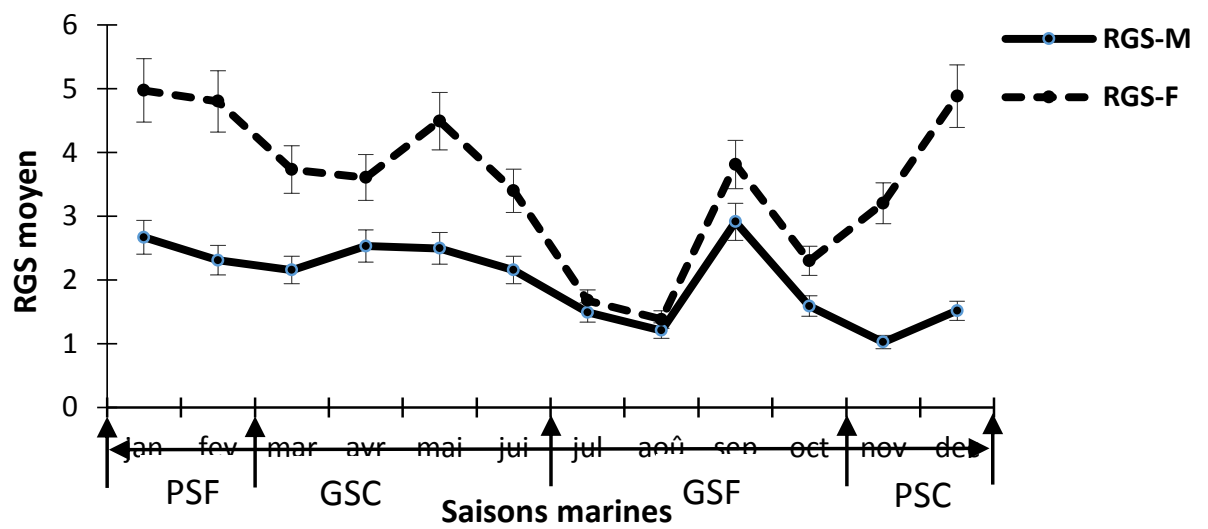

Figure 7. Evolution saisonnière du RGS chez l'auxide de janvier à décembre 2016

\section{Rapports Hépato-Somatiques}

L'évolution du RHS suit la même progression aussi bien chez les mâles que chez les femelles toute l'année. Deux phases sont observées : une ascendante et l'autre descendante. Le RHS décroit de janvier à février pour les deux sexes puis augmente jusqu'en mai, au cours de la grande saison chaude où il atteint un pic chez les femelles $(2,10 \pm 1,013)$. A partir de mai, il chute jusqu'en juillet et remonte en août dans la grande saison froide pour atteindre un pic chez les mâles $(1,60 \pm 0,286)$. Les valeurs de RHS les plus faibles sont observées en février au niveau des deux sexes avec des valeurs respectives de 0,88 $\pm 0,326$ (mâles) et de 1,01 $\pm 0,410$ (femelles). L'évolution saisonnière du RHS pour les mâles et femelles est maximale 
pendant la grande saison froide avec des valeurs respectives de $1,43 \pm 0,419$ (femelles) et de 1,29 $\pm 0,362$ (mâles).

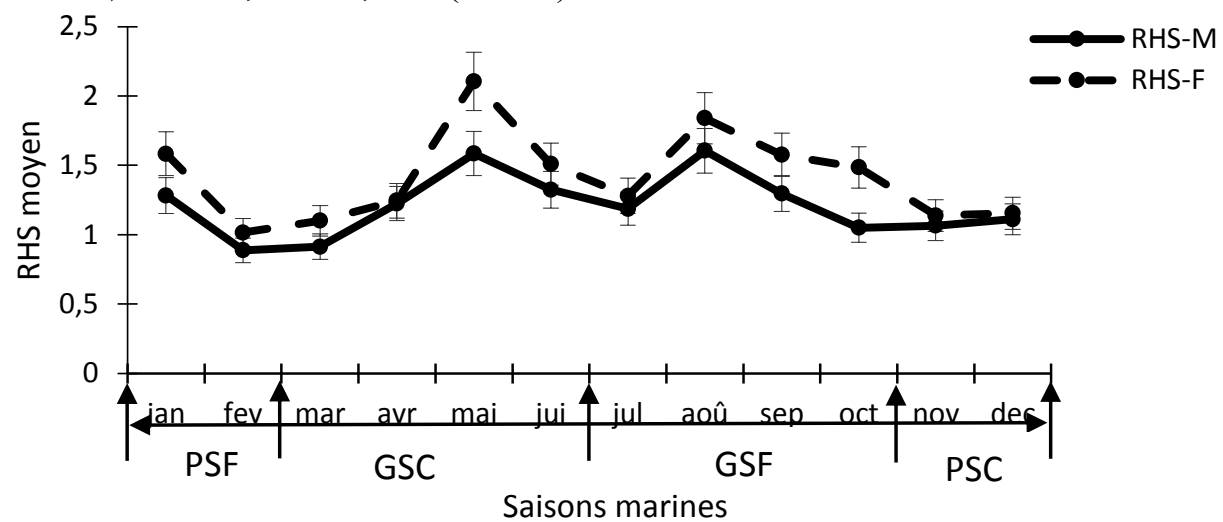

Figure 8. Evolution saisonnière du RHS chez l'auxide de janvier à décembre 2016.

\section{Facteur de Condition}

Les facteurs de condition de l'Auxis thazard mâle et femelle évoluent dans le même sens de janvier à décembre 2016. Ils présentent de légères variations aussi bien chez les femelles que chez les mâles. Chez les mâles, il oscille entre 1,48 $\pm 0,247$ et 1,88 $\pm 0,086$ avec une valeur moyenne de 1,76 \pm 0,148 . La plus petite valeur est observée au cours du mois de mai dans la grande saison chaude et la plus haute valeur dans le mois d'août pendant la grande saison froide. Pour les femelles, $\mathrm{K}$ varie de $1,51 \pm 0,300$ à $1,87 \pm$ 0,202 avec une moyenne de $1,79 \pm 0,150$. Les valeurs les plus basses et plus élevées sont respectivement observées dans le mois de mai pendant la grande saison chaude et au cours du mois de juillet dans la grande saison froide. Les chutes des valeurs de $\mathrm{K}$ observées correspondent aux maximums des RGS pour les deux sexes.

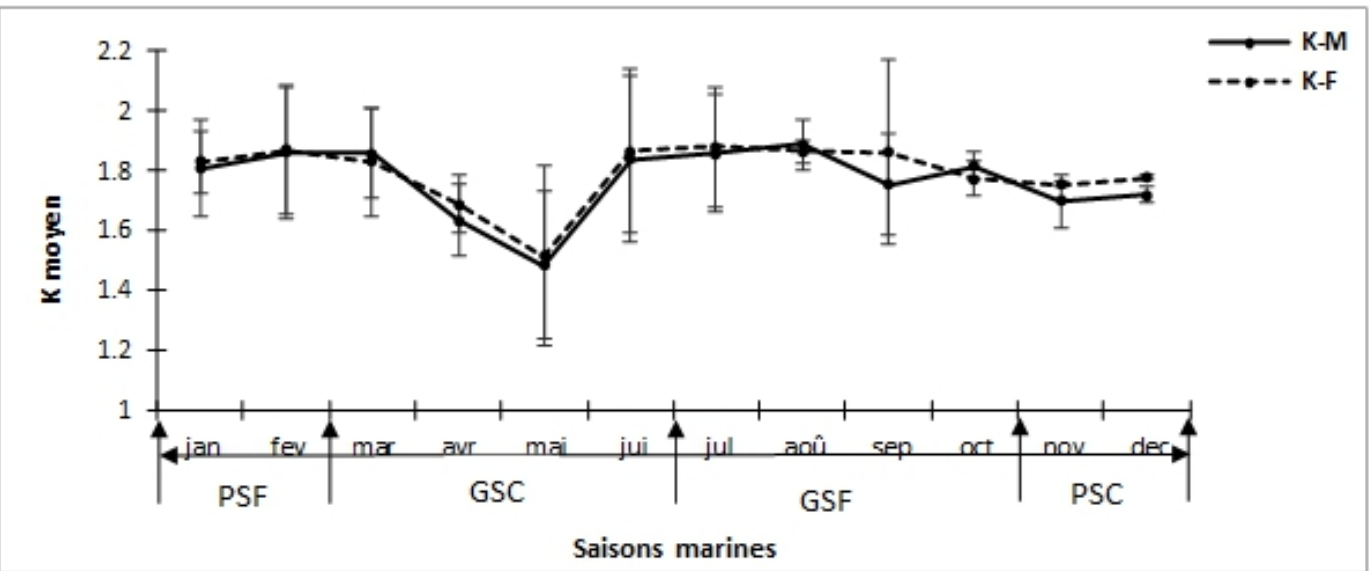

Figure 9. Evolution saisonnière du facteur de condition chez Auxis thazard de janvier à décembre 2016 


\section{Variations du RGS en Fonction des Stades de Maturité Sexuelle}

L'évolution du RGS chez les mâles et femelles en fonction des stades de maturité (figure 10) suit généralement la même progression. Le RGS augmente progressivement du stade 2 (immature) au stade 5 (ponte) puis baisse au stade 6 (post ponte). Les valeurs les plus faibles pour les mâles et femelles observées au stade 2 sont respectivement de 0,66 $\pm 0,716$ et $0,61 \pm$ 0,404 . Les valeurs les plus élevées quant à elles sont observées au stade 5 avec des valeurs de 2,96 $\pm 1,181$ (mâles) et 6,05 $\pm 2,196$ (femelles). La comparaison entre les RGS des différents stades aussi bien chez les femelles que chez les mâles (test HSD de Tukey) montre une différence significative $(\mathrm{p}<0,05)$.
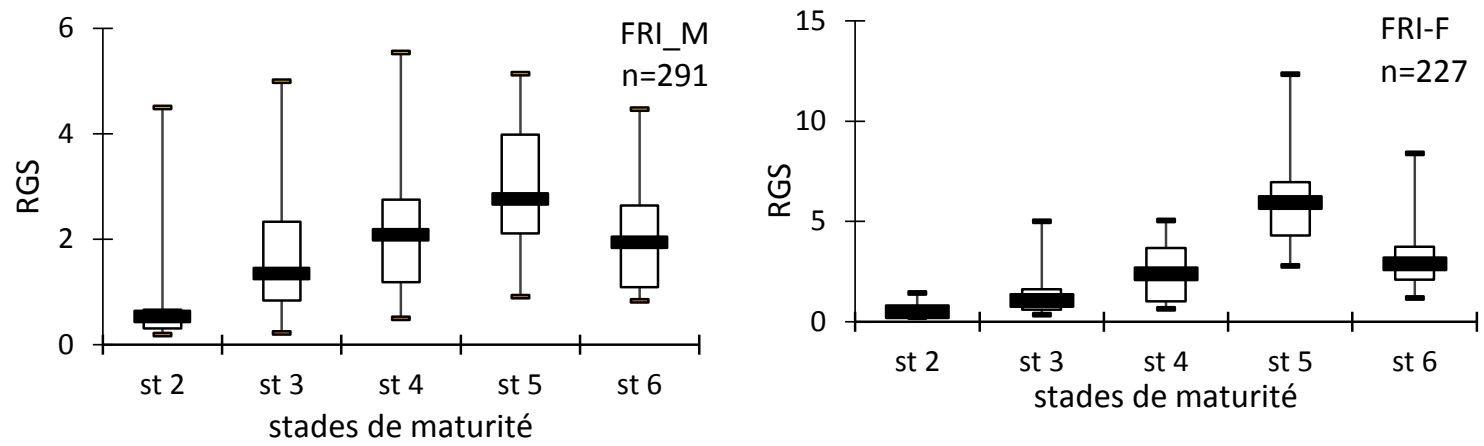

Figure 10. Variation du rapport gonado-somatique en fonction des stades de maturité sexuelle chez Auxis thazard de janvier à décembre 2016

\section{Variation du RHS en Fonction des Stades de Maturité Sexuelle}

L'évolution du RHS en fonction des stades de maturité chez l'auxide est similaire au niveau des deux sexes. Il croit du stade 2 (immature) au stade 4 (pré-ponte) pour chuter au stade 5 (ponte) et remonter légèrement au stade 6 (post-ponte). Le test HSD de Tukey appliqué aux moyennes des RHS pour les mâles et femelles ne montre aucune différence significative $(p>0,05)$.
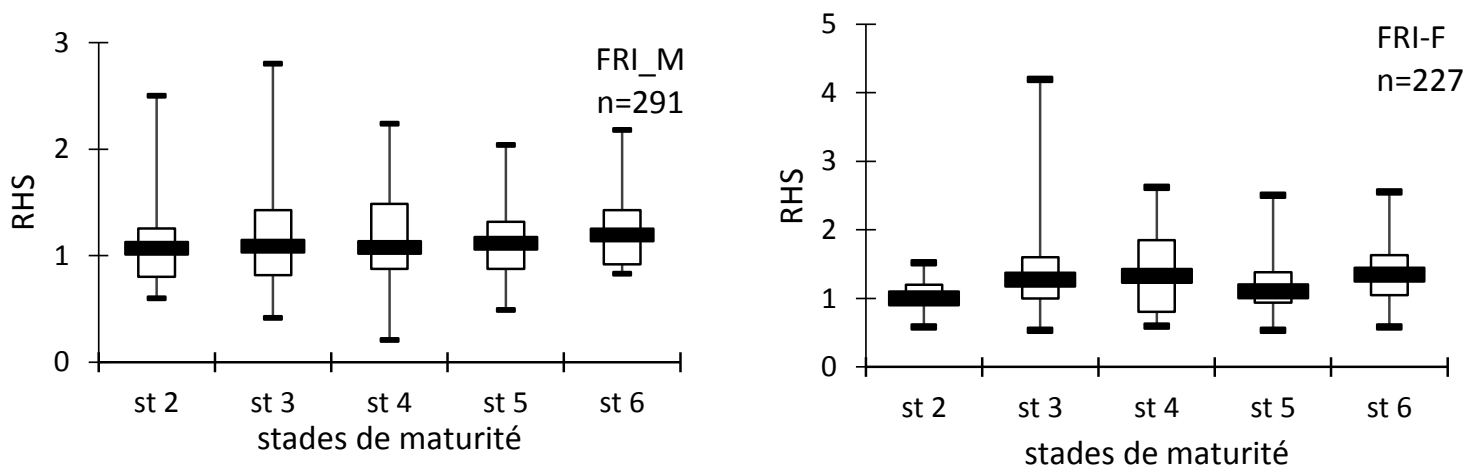

Figure 11. Variation du rapport hépato-somatique en fonction des stades de maturité sexuelle chez Auxis thazard de janvier à décembre 2016 


\section{Facteur de Condition en Fonction des Stades de Maturité}

L'évolution de K chez l'auxide tous sexes confondus en fonction des stades macroscopiques de la maturité sexuelle présente des variations du stade 2 au stade 6 . Il croit progressivement du stade 2 au stade 5 puis chute à partir du stade 6. La comparaison entre les moyennes de $\mathrm{K}$ aux différents stades de maturité (test HSD de Tukey) montre une différence significative à partir des stades 5 et $6(\mathrm{p}<0,05)$.
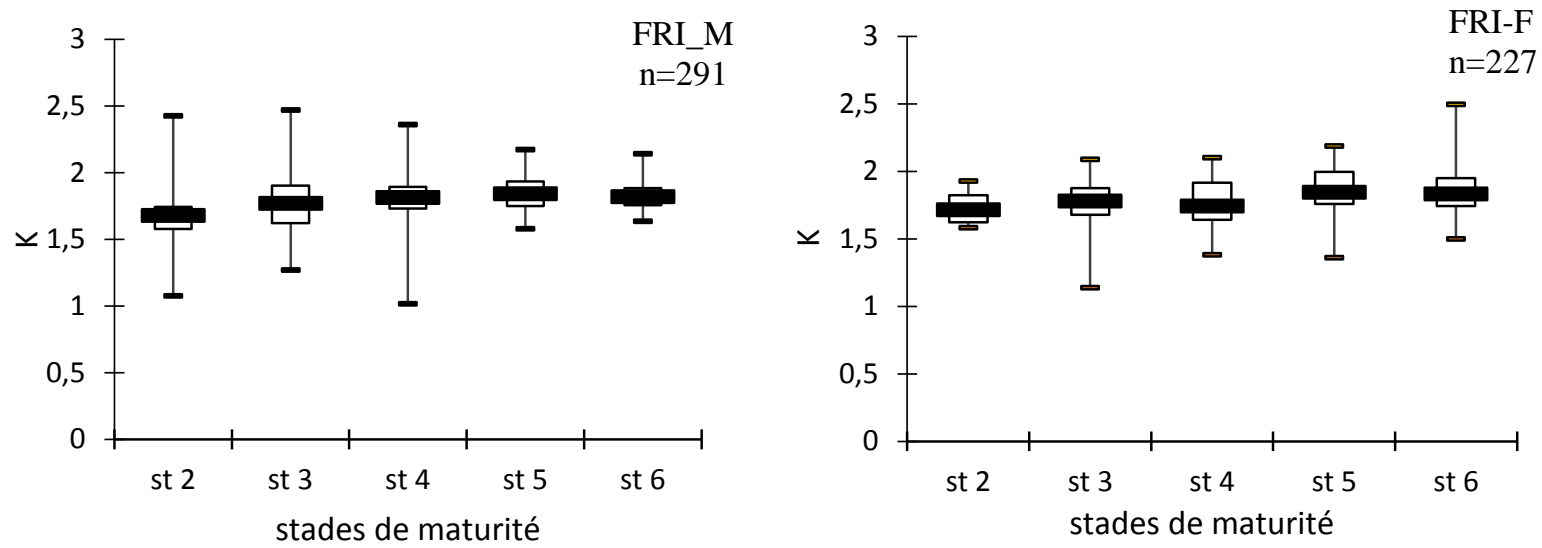

Figure 12. Variation du facteur de condition en fonction des stades de maturité sexuelle chez Auxis thazard de janvier à décembre 2016

\section{Relation Taille-Poids}

La taille des individus mâles varie de 26,5 à $46,3 \mathrm{~cm}$ et le poids oscille entre 200 et $1900 \mathrm{~g}$. Les femelles quant à elles, présentent une gamme de taille variant de 26 à $49 \mathrm{~cm}$ et des poids oscillant de 26 à $2450 \mathrm{~g}$. L'équation de relation taille-poids calculée chez les mâles est : $\mathrm{W}=0,0039$ $\mathrm{L}^{3,4108}\left(\mathrm{R}^{2}=0,93 ; \mathrm{N}=291\right)$. Chez les femelles, elle se présente comme suit : $\mathrm{W}=0,0036 \mathrm{~L}^{3,4376}\left(\mathrm{R}^{2}=0,91 ; \mathrm{N}=227\right)$, et au niveau des deux sexes combinés, l'on obtient : $\mathrm{W}=0,004 \mathrm{~L}^{3,4118}\left(\mathrm{R}^{2}=0,92 ; \mathrm{N}=519\right)$. Le test $\mathrm{T}$ de Student appliqué suivant le sexe montre une différence significative. La valeur du coefficient d'allométrie $\mathrm{b}$ déterminée est supérieure à la valeur théorique $(b=3)$ pour les mâles ( $t$-test, $b=3,4108, t=233,218, p<0,05)$, pour les femelles (t-test, $b=3,4376, t=227,776, p<0,05)$ et pour les deux sexes combinés ( $\mathrm{t}$-test, $\mathrm{b}=3,4118, \mathrm{t}=315,821, \mathrm{p}<0,05$ ). Le coefficient $\mathrm{b}$ des femelles est supérieur à celui des mâles (bf $\left.=3,4376>b_{m}=3,4108\right)$. 

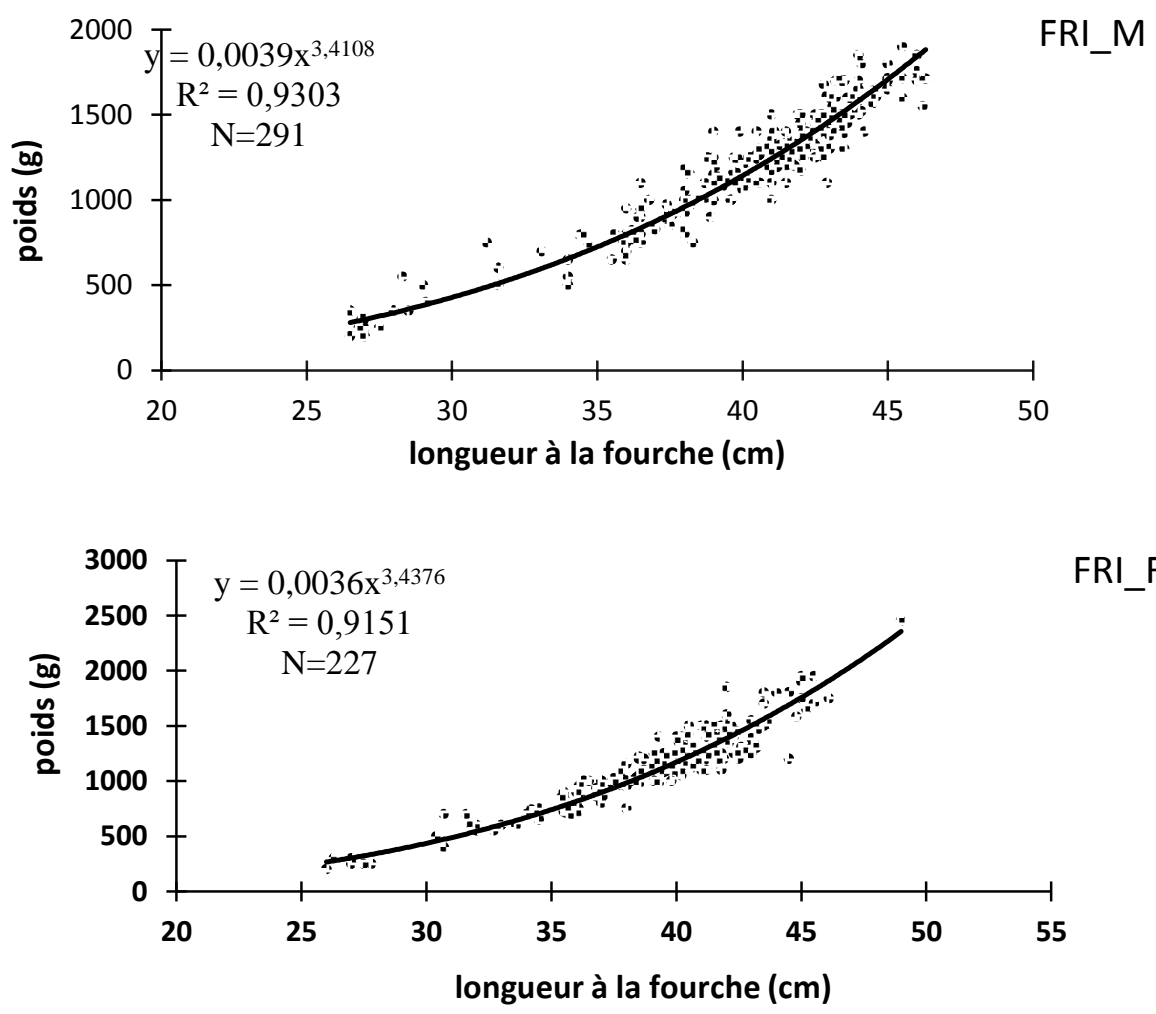

FRI_F

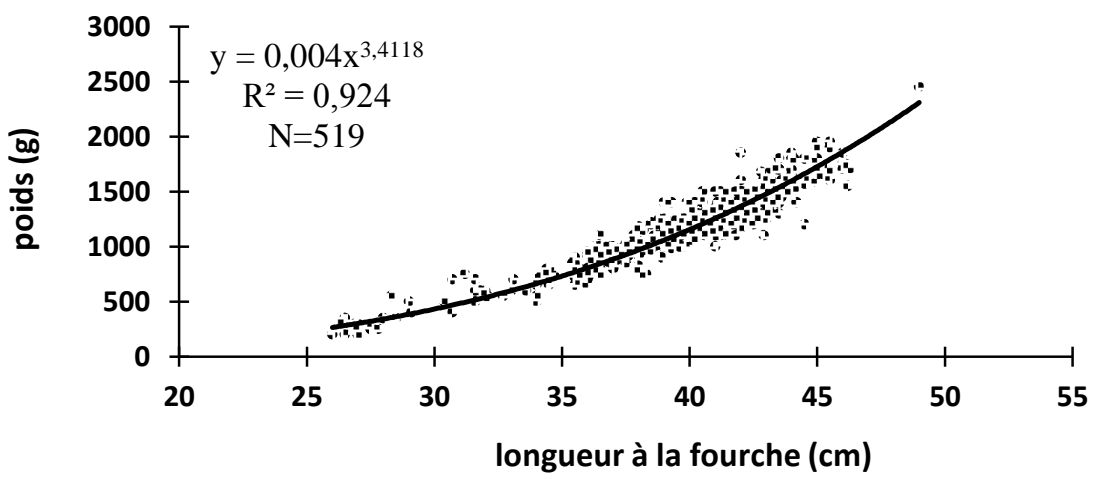

Figure 13. Relation Taille-poids l'auxide mâle (FRI_M), femelle (FRI_F) et population (FRI_P) de la pêcherie artisanale de Côte d'Ivoire de janvier à décembre 2016

\section{Discussion}

La sex-ratio globale déterminée pour l'ensemble de l'échantillon a montré une prédominance des mâles. Cependant, sur la totalité des quatre saisons marines, seule la petite saison chaude (novembre-décembre) a enregistré une différence significative au niveau des sexes avec une 
proportion élevée des spécimens mâles. En effet, l'inégale répartition ou la dominance de l'un des sexes est un phénomène couramment observé chez de nombreuses espèces de poissons téléostéens (Tiehoua, 2016). Cette situation s'expliquerait par le fait que chez ces poissons, plusieurs facteurs à savoir le déplacement pour la quête de la nourriture, la reproduction, la croissance différentielle et le taux de mortalité par sexe influenceraient la distribution spatio-temporelle des sexes chez ces espèces (Mellinger, 2002). Ces résultats sont en accords avec ceux obtenus sur Auxis thazard dans les eaux indiennes par Muthiah (1985) qui note une prédominance des femelles. Ils sont aussi similaires à ceux de Macias et al. (2005), observés sur une autre espèce du même genre (Auxis rochei) dans l'ouest de la méditerranée espagnole. Pour les trois autres saisons (PSF, GSC, GSF), les sex-ratios déterminées n'étaient pas différentes de la valeur théorique. Cette parité entre les sexes a été notée chez Auxis rochei au niveau de la méditerranée en Turquie par Abdullah et al. (2010).

L'analyse de l'évolution saisonnière des stades macroscopiques de la maturité sexuelle, du RGS combinée au RHS suggèrent que Auxis thazard se reproduit au moins une fois dans les quatre saisons de l'année à savoir la petite saison froide, la grande saison chaude, la grande saison froide et la petite saison chaude. En effet, l'activité reproductrice de l'auxide semble être continue du fait que les femelles matures sont rencontrées dans toutes les saisons de l'année. Le développement maximum des gonades des poissons est atteint au mois de décembre dans la petite saison chaude, au mois de janvier dans la petite saison froide, au mois de mai dans la grande saison chaude et au mois de septembre dans la grande saison froide. La chute du poids des gonades observée à partir des mois de février (PSF), juin (GSC) et octobre (GSF) correspondent aux périodes maximales de reproduction de l'auxide dans le golfe de Guinée.

La reproduction étalée sur toute l'année pour cette espèce s'explique par le fait que la maturation chez les poissons se fait très rapidement du stade 3 au stade 6 et qu'après avoir pondu, ils peuvent soit entrer en repos sexuel (retour au stade 2) ou soit entamer aussitôt une nouvelle maturation (début stade 4); le second cas de figure est celui observé chez Auxis thazard (Cayré, 1985). Ces résultats sont similaires à ceux de l'Iccat (2010) sur Auxis rochei, qui stipulent que la reproduction chez l'espèce a lieu de février à novembre dans l'océan atlantique. Ils sont par contre différents de ceux de Bahou et al. (2016) enregistrés sur les femelles de Auxis thazard dans les eaux ivoiriennes. Selon cet auteur, l'espèce ne se reproduirait qu'une seule fois dans l'année et au cours de la grande saison froide.

La comparaison des courbes d'évolution mensuelle des RGS et des RHS montre que ces deux indices progressent presque de la même manière. Les pics des RGS correspondent aux pics des RHS. Ce qui traduirait que 
l'augmentation du poids du foie est liée à l'accroissement de l'activité génitale. Cet état de fait s'explique par le fait qu'Auxis thazard serait un poisson gras dont la caractéristique principale est de stocker les réserves d'énergie essentiellement dans les muscles, dans les mésentères péri viscéraux et sous la peau. Ces réserves passent ensuite dans le foie et dans les gonades pour assurer les besoins énergétiques en période de reproduction, d'où la faible valeur du facteur de condition constatée pendant la période de reproduction (Djadji et al., 2013). Ces résultats sont confirmés par Bahou et al. (2016) qui notent une évolution synchrone entre le RGS et le RHS, puis une baisse du facteur de condition pendant la reproduction chez les femelles d'auxide.

L'évolution du RGS et du RHS en fonction des stades de maturité montrent deux phases : une évolution similaire des deux rapports du stade 2 au stade 4, puis une évolution différente à partir du stade 5. Cette baisse du RHS et l'augmentation du RGS au stade 4 traduisent le transfert de l'énergie contenue dans le foie vers les gonades pour assurer les fonctions de la reproduction (Belhoucine, 2012).

La relation taille-poids déterminée pour l'espèce montre un coefficient d'allométrie supérieur à la valeur théorique au niveau des deux sexes. Ces résultats révèlent que l'auxide a une meilleure croissance en poids qu'en longueur, donc présente une allométrie majorante. Nos résultats sont semblables à ceux de Ramos et al. (1985) obtenus sur Auxis rochei dans la méditerranée et ceux de Bahou et al. (2016) sur les femelles de Auxis thazard dans le golfe de Guinée. Par contre, ils diffèrent de ceux de Macias et al. (2005) sur Auxis rochei dans la méditerranée.

Pour les deux sexes séparés, il est noté que le coefficient d'allométrie des femelles est supérieur à celui des mâles. Ce qui suggère que les femelles ont une meilleure croissance en poids que les mâles. En effet, pour des spécimens ayant un même stade de maturité sexuelle et une même taille, il a été noté qu'en raison de l'augmentation importante du poids des gonades femelles (ovaires) que ceux des mâles (testicules), les femelles présenteraient un poids plus élevé que les mâles chez les thonidés (Hajjej et al., 2012). Certes, le sexe est l'un des principaux facteurs qui affecte la relation taillepoids, mais il en existe d'autres tels que la température, la salinité, le stade de maturité et l'abondance de la nourriture (Andrade \& Campos, 2002).

\section{Conclusion}

Le suivi de la variation saisonnière de certains paramètres de la reproduction chez Auxis thazard montre que cette espèce se reproduit dans toutes les saisons de l'année mais à des degrés d'intensité différente dans le golfe de Guinée. Cette espèce présente une meilleure croissance en poids 
qu'en longueur. Au sein de cette population, il a été noté que les femelles ont un gain de poids qui semble plus important que chez les mâles.

\section{References:}

1. Abé, J., Kouassi, A. M., Ibo, G. J., N'guessan, N., Kouadio, A., N'goran, Y. N. \& Kaba, N.

2. (2002). Côte d'Ivoire coastal zone. phase 1: integrated environmental problem analysis.

3. National report of GEF MSP Sub-Saharan Africa Project (GF/60100016): "Development and Protection of the Coastal and Marine Environment in Sub-Saharan Africa"(76 p).

4. Abdullah, E. K., Didem, G., Erol, R. B., Tülay, A. \& Saadet, K. F. (2010). Some reproductive aspects of female bullet tuna, Auxis rochei (Risso), from the Turkish Mediterranean coasts: African Journal of Biotechnology Vol. 9(40), (pp. 6813-6818).

5. Andrade, H. A. \& Campos, R. O. (2002). Allometry coefficient variations of the length-weight relationship of skipjack tuna (Katsuwonus pelamis) caught in the southwest Atlantic: Fisheries Research, 55( 307-312pp).

6. Bahou, L., Atsé B. C., D’Almeida, M-A. \& Tidiani, K. (2016). Reproductive biology of Female frigate tuna Auxis thazard (Lacepède, 1800) caught in Coastal marine waters of Côte d'Ivoire: Vol. 5, Issue 4, April 2016 (16 p).

7. Belhoucine, F. (2012). Etude de la biologie de la croissance et de la reproduction d'un poisson téléostéen le merlu et son utilisation comme indicateur biologique de la pollution par les métaux lourds dans la baie d'Oran (Algérie) : Thèse de doctorat (325 p).

8. Diaha, N. C., N'da, K. \& Kouassi, K. D. (2009). Etude comparée de la pêche des thonidés

9. mineurs par les chaluts doubles et les pirogues dans la Zone Economique Exclusive (ZEE) ivoirienne : Tropicultura, 27 (3) : 152 158.

10. Djadji, G. L., Atse, B. C., Sylla, S., Konan, K. J., Kouassi, N. J. (2013). Reproduction du Mugilidae Mugil cephalus Linné, 1758 dans deux complexes lagunaires (lagunes Ébrié et de Grand-Lahou) de la Côte d'Ivoire: International Journal of Biologie and Chemical Sciences 7(4) (pp 1701-1716).

11. Hajjej, G., Hattour, A., Hajjej, A., Cherif, M., Allaya, H., Jarboui, O. \& Bouain, A. (2012). Age and growth of little tunny, Euthynnus alletteratus (Rafinesque, 1810), from the Tunisian Mediterranean coasts: Cah.Biol.Mar (2012) 53(113-122. 10 p). 
12. Le Cren, E. D. (1951). The length-weight relationship and seasonal cycle in gonad weight and condition in the Perch (P. fluviatilis): Journal of Animal Ecology 20: (201-219p).

13. Macías, D., Gómez-Vives M. J., \& de la Serna1 J. M. (2005). Some reproductive aspects of bullet tuna (Auxis rochei) from the south western spanish Mediterranean: Col. Vol. Sci. Pap. ICCAT, 58(2) (484-495, $12 \mathrm{p})$.

14. Manuel Iccat (2010). 1ère édition (janvier 2010) : (8 p).

15. Mellinger, J. (2002). Sexualité et reproduction des poissons : Paris, CNRS Editions (349 p).

16. Micha, J. C. (1973). Etude des populations piscicoles de l'Ubangui et tentatives de sélection et d'adaptation de quelques espèces à l'étang de pisciculture : Centre Technique Forestier Tropical, Nogent-surMarne, France, (110 p).

17. Muthiah, C. (1985). Fishery and bionomics of tunas at Mangalore: CMFRI Bull., 36: 51-70.

18. Pezennec, O. \& Bard, F.X. (1992). Importance écologique de la petite saison d'upwelling Ivoiro-Ghanéenne et changements dans la pêcherie de Sardinella aurita: Aquat. Living Resour. 5, (249-259p).

19. Ramos A., A lot E., \& Camiñas, J. A. (1985). Relación talla/peso de la melva, Auxis thazard, para el Atlántico y Mediterráneo : Collect. Vol. Sci. Pap. ICCAT, 25: 265-268 (1986).

20. Rey, J. C., A lot, E. \& Ramos, A. (1984). Sinopsis biologica del bonito, Sarda (Boch), del Mediterraneo y Atlantico Este: ICCAT Coll. Vol. Sci. Pap., XX(2): (46-502p).

21. Ricker, W. E. (1975). Computation and interpretation of biological statistics of fish population: Bull of the Fisheries Research Board of Canada (pp. 191, 382).

22. Ricker, W. E. (1980). Calcul et interprétation des statistiques biologiques des populations de poissons: Bulletin of Fisheries Research Board, Canada 32: 1369-1381.

23. Robinson, M. S., Athony, T. R., Littau, S. R., Herckes, P., Nelson, X., Poplin, G. S. \& Burges, J. L. (2008). Occupation PAH exposures during preseribed pile.burns : Ann.Occ.Hyg. 52(6): (497-508).

24. Tiéhoua, K., Kouakou F. K. I., Konan K. S. \& N'Da, K., (2016). Biologie de la reproduction du Sciaenidae pseudotolithus elongatus dans la lagune Ebrié (Cote d'Ivoire): European Scientific Journal February 2016 edition vol.12, No.6 ISSN: 1857 - 7881 (Print) e ISSN 1857- 7431. (19 p).

25. West, G. (1990). Methods of assessing ovarian development in fishes: a review. Australian Journal of Marine and Freshwater Research, 41: (pp. 199-222). 